\title{
Value of plasma B type natriuretic peptide measurement for heart disease screening in a Japanese population
}

\author{
M Nakamura, H Endo, M Nasu, N Arakawa, T Segawa, K Hiramori
}

Heart 2002;87:131-135

See end of article for authors' affiliations

Correspondence to:

Dr M Nakamura, Second

Department of Internal

Medicine, Iwate Medical

University, 19-1 Uchimaru,

Morioka, Iwate

020-8505, Japan;

nkmrmoto@iwate-med.ac.jp

Accepted

8 October 2001

\begin{abstract}
Background: Conflict exists regarding the usefulness of measuring plasma B type natriuretic peptide (BNP) concentrations for identifying impaired left ventricular (LV) systolic function during mass screening. Various cardiac abnormalities, regardless of degree of LV dysfunction, are prone to carry a high risk of cardiovascular events.

Objective: To examine the validity of plasma BNP measurement for detection of various cardiac abnormalities in a population with a low prevalence of coronary heart disease and LV systolic dysfunction.

Design and setting: Participants in this cross sectional study attended a health screening programme in Iwate, northern Japan. Plasma BNP concentrations were determined in 1098 consecutive subjects (mean age 56 years) by direct radioimmunoassay. All subjects underwent multiphasic health checkups including physical examination, ECG, chest radiography, and transthoracic echocardiography.

Results: Conventional diagnostic methods showed 39 subjects to have a wide range of cardiac abnormalities: lone atrial fibrillation or flutter in 11; previous myocardial infarction in seven; valvar heart disease in seven; hypertensive heart disease in six; cardiomyopathy in six; atrial septal defect in one; and cor pulmonale in one. No subjects had a low LV ejection fraction $(<40 \%)$. To assess the utility of plasma BNP measurement for identification of such patients, receiver operating characteristic analysis was performed. The optimal threshold for identification was a BNP concentration of $50 \mathrm{pg} / \mathrm{ml}$ with sensitivity of $89.7 \%$ and specificity of $95.7 \%$. The area under the receiver operating characteristic curve was 0.970 . The positive and negative predictive values at the cutoff level were $44.3 \%$ and $99.6 \%$, respectively.

Conclusion: Measurement of plasma BNP concentration is a very efficient and cost effective mass screening technique for identifying patients with various cardiac abnormalities regardless of aetiology and degree of LV systolic dysfunction that can potentially develop into obvious heart failure and carry a high risk of a cardiovascular event.
\end{abstract}

$\mathrm{T}$ he prevalence of congestive heart failure may continue to rise as the proportion of elderly people within the population increases. ${ }^{1}$ Although the prognosis for this disorder may have improved with recent medical and surgical interventions, the expected duration of survival has been reported to remain less than two years. ${ }^{2}$ To make a major impact on the prognosis for this disorder, it would be important to recognise various forms of heart disease early enough to limit or interrupt the progression of the condition before the occurrence of overt heart failure. ${ }^{3}$ However, no simple screening test without the need for specialised techniques has yet been established for this condition.

$\mathrm{B}$ type natriuretic peptide (BNP) is synthesised and released from myocardium in response to an increase in ventricular filling pressures. ${ }^{4-6}$ Plasma BNP concentrations have been reported to be increased in various forms of heart disease including cardiomyopathy, ${ }^{78}$ hypertensive heart disease, ${ }^{9}$ valvar heart disease, ${ }^{4}$ and atrial fibrillation..$^{10}$ Some studies have shown that measurement of plasma BNP concentrations is useful for detecting patients with impaired left ventricular (LV) systolic function in hospital and community based settings. ${ }^{11-14}$ However, in elderly patients taking diuretics for congestive heart failure, BNP testing is not useful for differentiating those with LV systolic dysfunction (defined as having an LV ejection fraction $<30 \%$ ) from those with a normal ejection fraction. ${ }^{15}$ Similarly, in patients with previous myocardial infarction, plasma BNP measurement is unable to distinguish between patients with mild LV systolic dysfunction and those with preserved LV systolic function. ${ }^{16}$ In one community based study the sensitivity of BNP measurement for detecting impaired LV systolic function without cardiac hypertrophy was reported to be $<30 \% .{ }^{17}$ In addition, the positive predictive value of BNP testing for detection of LV dysfunction has been reported to be relatively low. ${ }^{11}{ }^{12}$ These observations suggest that circulating concentrations of BNP may be increased by factors other than impaired LV systolic function, ${ }^{18}$ including mild LV dysfunction, ventricular volume load, and LV hypertrophy. We therefore speculated that plasma BNP measurement may be useful in mass screening for a wide range of cardiac abnormalities, especially precursors of heart failure, in a population with a low prevalence of LV systolic dysfunction.

In an earlier study we reported that measuring plasma BNP was useful for the selection of subjects with various forms of heart disease in multiphasic health screening. ${ }^{19}$ This study suggested that plasma BNP measurement is valid for detecting subjects with previous myocardial infarction, cardiomyopathy, hypertensive heart disease, atrial fibrillation, or valvar heart disease. All of these disorders are important precursors of heart failure and generate a high prevalence of cardiovascular events regardless of aetiology and degree of LV dysfunction. However, these potentially serious outcomes may be preventable, or at least deferred, by medical or surgical intervention. This earlier study suffered from the shortcoming

Abbreviations: $\mathrm{BNP}, \mathrm{B}$ type natriuretic peptide; $\mathrm{Cl}$, confidence interval; $\mathrm{LV}$, left ventricular; MONICA, monitoring trends and determinants in cardiovascular disease 
of a lack of complete echocardiographic data on LV systolic function, LV geometry, and valve regurgitation. As a consequence, a number of subjects with mild LV dysfunction, LV hypertrophy, and valvar heart disease may have missed detection. To validate our hypothesis that plasma BNP measurement is an efficient blood test for detection of various types of heart disease, especially precursor forms of heart failure, the present study incorporated transthoracic echocardiography on all subjects in conjunction with ECG and chest radiography.

\section{METHODS}

\section{Subjects}

The patient sample comprised 1110 consecutive subjects (701 men, 409 women; mean age 55.8 years, range $25-83$ years) who attended a multiphasic health checkup programme run by the Iwate Health Maintenance Association (IHMA) between June 1998 and August 2000. IHMA is a non-profit health maintenance organisation that covers more than half the population in a rural area of the Iwate prefecture. Most of the subjects were recruited through a Japanese agricultural cooperative association in northern Iwate prefecture. Health checkups were performed in response to a circular or a home visit invitation by health care personnel. Participation was voluntary and the sample was thus deemed to be representative of the local population, except for the extremes of wealth and poverty. There were no limitations on participant selection even if they had any history of heart disease and were receiving ongoing medications, but hospital inpatients were not included in the study population.

\section{Measurements}

All subjects underwent physical examination, chest radiography, supine 12 lead ECG, and blood pressure measurement. Blood tests were also taken for blood cell count, liver and renal function, and glucose and lipid concentrations. These samples were obtained during the morning of attendance and were taken from a forearm vein with the patient in a sitting position. While these standard samples were being taken, an additional $2 \mathrm{ml}$ sample of venous blood was collected into a test tube containing EDTA-2Na for plasma BNP measurement. Blood samples was centrifuged immediately and the plasma was stored at $-80^{\circ} \mathrm{C}$ until the BNP assay was carried out.

Plasma BNP concentrations were measured by direct radioimmunoassay using monoclonal antibodies specific for human BNP (Shiono RIA BNP kit, Shionogi \& Co, Ltd, Osaka, Japan). Cross reactivity of the antibody was $100 \%$ for human BNP and $0.001 \%$ for human atrial natriuretic peptide. The intra-assay and interassay coefficients of variation were $5 \%$ and $6 \%$, respectively. The assay was performed within three months of sampling.

Echocardiography was performed with a high performance Acuson 512 Sequoia (Acuson, Mountain View, California, USA) with a 3V2C transducer (3.5 MHz with harmonic image). Of the data obtained, 1098 of 1110 (99\%) were analysable for left atrial dimension, LV septal and posterior wall thickness, and LV chamber dimensions, which were measured according to guidelines of the American Society of Echocardiography. ${ }^{20} \mathrm{LV}$ ejection fraction was calculated by Teichholz's rule. ${ }^{21}$ The echocardiographic report was compiled immediately after the test by an echocardiography technician of IHMA, with video recorded figures being checked after this by an experienced echocardiogram specialist (Ma N). The median variation between two readings by the same observer or by different observers for LV ejection fraction or LV dimensions was within $10 \%$ in both cases. These procedures were performed with no knowledge of the plasma BNP concentrations.

\section{Diagnosis}

Diagnosis was carried out by two independent cardiologists $(\mathrm{H}$ E and Mo $\mathrm{N})$ on the basis of each subject's medical questionnaire, chest radiograph, ECG, and echocardiographic report. Again, this was done with no knowledge of plasma BNP concentrations. "Hypertensive heart disease" was diagnosed if subjects had echocardiographically documented LV hypertrophy ( $\geqslant 13 \mathrm{~mm}$ ) and ST/T segment changes on ECG with increased blood pressure (systolic blood pressure $\geqslant 140 \mathrm{~mm} \mathrm{Hg}$ or diastolic blood pressure $\geqslant 90 \mathrm{~mm} \mathrm{Hg}$ ) or were taking antihypertensive drugs. "Mitral or aortic valve regurgitation" was defined as a regurgitation grade $>3^{\circ}$ with a significant increase in LV chamber size. When subjects with no current or previous hypertension had echocardiographically documented LV hypertrophy ( $\geqslant 13 \mathrm{~mm}$ ) with giant negative $\mathrm{T}$ wave in at least four leads on ECG, they were designated as having "hypertrophic cardiomyopathy."

\section{Statistical analysis}

Receiver operating characteristic (ROC) curves were constructed to assess the sensitivity and specificity of plasma BNP throughout the range of concentrations as an indicator of each disease category. The area under the curve and 95\% confidence interval (CI) of each ROC curve were calculated to provide a measure of the overall diagnostic accuracy of the test. The optimum concentration of BNP for calculation of positive and negative predictive values was obtained from the ROC analysis.

\section{RESULTS}

Table 1 shows the clinical characteristics of the study subjects. Of the total sample, 293 subjects were regarded as hypertensive as defined by systolic blood pressure $>140 \mathrm{~mm} \mathrm{Hg}$ or diastolic blood pressure $>90 \mathrm{~mm} \mathrm{Hg}$. Of this group, 19l received regular antihypertensive medication while the remaining 102 were not treated. This prevalence rate of hypertension is comparable with that reported previously in a sample from a rural area of northern Japan. ${ }^{22}$ Eighteen subjects were prescribed drugs for hypercholesterolaemia and $67 \mathrm{sub}-$ jects had serum total cholesterol concentrations $>6.2 \mathrm{mmol} / \mathrm{l}$. Sixty one subjects showed mildly increased serum creatinine ( $>0.115 \mathrm{mmol} / \mathrm{l}$ ) but none were on haemodialysis. Abnormal ECG findings were observed in 301 subjects $(27.4 \%)$, including left or right bundle branch block, ST/T segment changes, voltage elevation for LV hypertrophy, premature beats, or atrial fibrillation or flutter. However, by transthoracic echocardiography, no subjects showed LV systolic dysfunction (LV ejection fraction $<40 \%)$. Several subjects $(n=8)$ reported

Table 1 Clinical characteristics and documented heart disease in 1098 examined subjects

\begin{tabular}{lll} 
& $\begin{array}{c}\text { Mean or } \\
\text { number }\end{array}$ & Range \\
\hline Mean age (years) & 55.8 & $25-83$ \\
Sex (male) & 693 & $(63.1 \%)$ \\
Hypertension & 293 & $(26.7 \%)$ \\
Hypercholesterolaemia & 85 & $(7.7 \%)$ \\
ECG abnormality & 301 & $(27.4 \%)$ \\
Echocardiographic data & & \\
Mean LV ejection fraction (\%) & 69.5 & $45-87$ \\
Mean LV posterior wall thickness (mm) & 9.5 & $5-15$ \\
Mean LV diastolic dimension (mm) & 47.1 & $32-62$ \\
Documented heart disease & 11 & \\
Lone atrial fibrillation or flutter & 7 & \\
Previous myocardial infarction & 7 & \\
Valvar heart disease & 6 & \\
Hypertensive heart disease & 5 & \\
Hypertrophic cardiomyopathy & 1 & \\
Dilated cardiomyopathy & 1 & \\
Atrial septal defect & 1 & \\
Cor pulmonale & $3.6 \%)$ \\
Total & 3 & \\
\hline LV, left ventricular & & \\
\hline
\end{tabular}




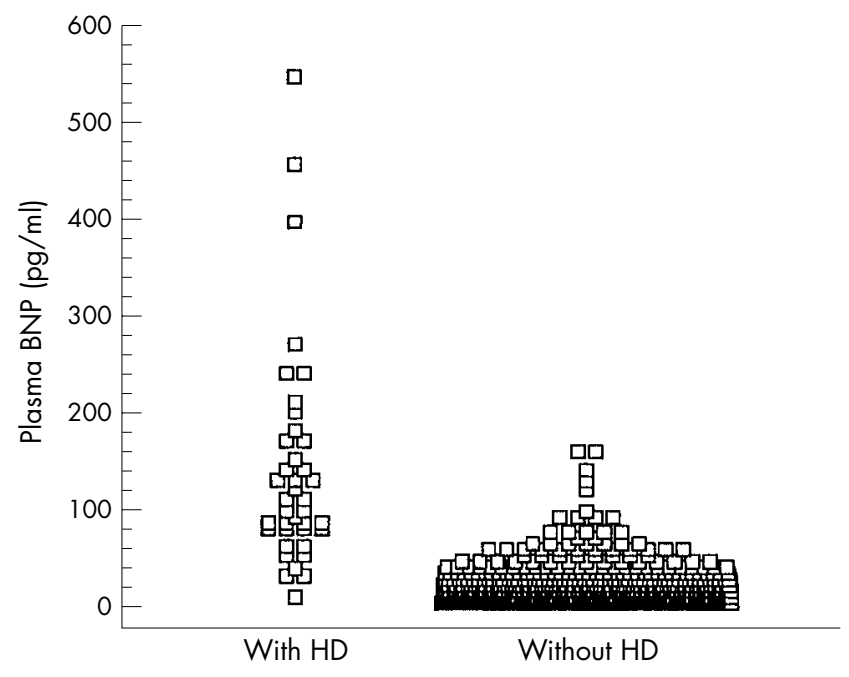

Figure 1 Distribution of plasma concentrations of B type natriuretic peptide (BNP) in subjects with and without heart disease (HD).

the use of anti-angina medication for a history of angina like pain. However, since detailed information such as results of stress testing or coronary angiography could not be obtained, these subjects were excluded from the ROC analysis.

Thirty nine subjects out of 1098 (3.6\%) had some form of heart disease from various aetiologies (table 1). The diagnoses were lone atrial fibrillation or flutter in 11 , previous myocardial infarction in seven, valvar heart disease in seven, hypertensive heart disease in six, hypertrophic cardiomyopathy in five, dilated cardiomyopathy in one, atrial septal defect in one, and cor pulmonale in one subject.

In their medical questionnaire, 12 of 39 subjects (31\%) listed one or more cardiovascular comorbidity, mainly hypertension. Eighteen of the respondents with heart disease $(46 \%)$ were not on any medical treatments. Five of 11 patients with lone atrial fibrillation or flutter did not describe any of their previous medical care. Four of seven patients with previous myocardial infarction had a history of myocardial infarction, while the remaining three patients had no history of the disease. Valvar heart disease had previously been diagnosed in only one patient with this disorder and four patients in this category were treated as having hypertension or "arrhythmia". Three patients with hypertensive heart disease did not receive any medical care including antihypertensive treatment. Three patients with hypertrophic cardiomyopathy did not have a medical history of this disorder.

Figure 1 shows the distribution of plasma BNP concentrations in subjects with and without heart disease. In subjects without heart disease, more than 95\% had plasma BNP concentrations of $<50 \mathrm{pg} / \mathrm{ml}$, whereas among those with some form of heart disease, approximately $90 \%$ had plasma BNP concentrations $>50 \mathrm{pg} / \mathrm{ml}$.

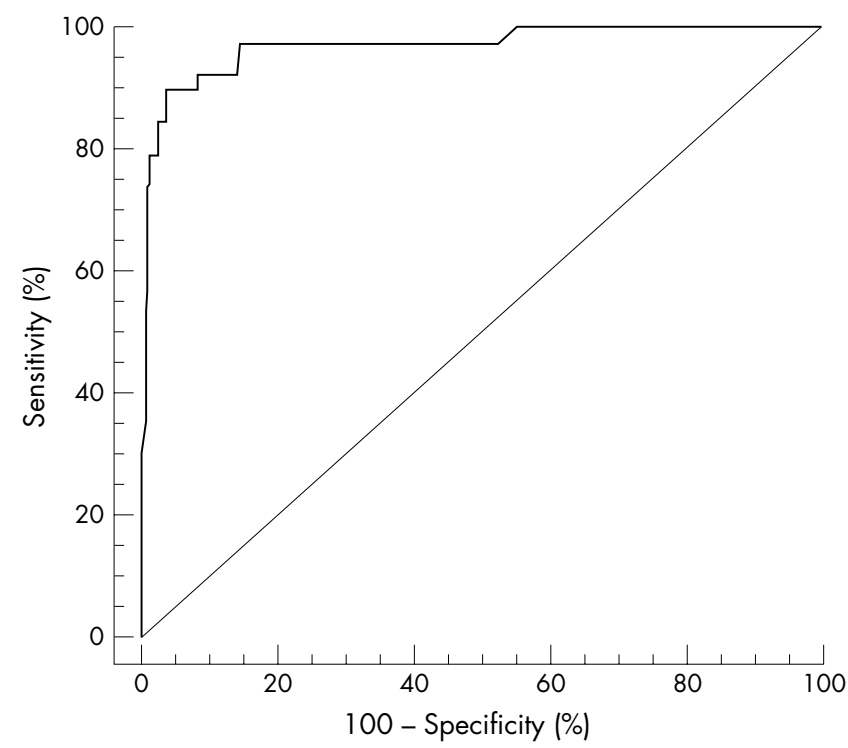

Figure 2 Receiver operating characteristic curve of the ability of plasma B type natriuretic peptide concentration to predict precursors of heart failure (previous myocardial infarction, lone atrial fibrillation or flutter, valvar heart disease, hypertensive heart disease, cardiomyopathy, adult type congenital heart disease, and cor pulmonale). The area under the receiver operating characteristic curve was 0.970 . The optimal threshold for detection of these subjects was $50 \mathrm{pg} / \mathrm{ml}$ with sensitivity of $89.7 \%$ and specificity of $95.7 \%$.

The highest degree of diagnostic accuracy in plasma BNP measurement was shown to be previous myocardial infarction (table 2), with sensitivity and specificity of $100 \%$ and $97 \%$, respectively. The area under the ROC curve was 0.986 (95\% CI 0.977 to 0.992 ). Identically high levels of sensitivity and similar diagnostic accuracy were obtained for lone atrial fibrillation or flutter, hypertensive heart disease, and valvar heart disease. In comparison, plasma BNP measurement was significantly less useful for the detection of hypertrophic cardiomyopathy ( $\mathrm{p}<0.05 v$ the above four disease categories).

The overall power of plasma BNP to detect these conditions $(\mathrm{n}=39)$ was very high. The optimal threshold was $50 \mathrm{pg} / \mathrm{ml}$ with sensitivity of $89.7 \%$ and specificity of $95.7 \%$ (fig 2 ) and an area under the ROC curve of 0.970 (95\% CI 0.958 to 0.979 ). The positive and negative predictive values at the cutoff were $44.3 \%$ and $99.6 \%$, respectively.

The ability of ECG to detect heart disease was compared with that of the BNP test. All selected heart disease subjects showed apparent ECG abnormalities. In the remaining subjects without heart disease, 272 subjects showed ECG abnormalities. Thus, the sensitivity and specificity of ECG for the detection of heart disease were $100 \%$ and $74 \%$, respectively. The positive predictive value for ECG testing was $12.5 \%$. The area under the ROC curve for ECG testing was significantly less than that for BNP testing $(0.873 v 0.987 ; \mathrm{p}<0.01)$.

Table 2 Diagnostic value of plasma B type natriuretic peptide concentration in detecting several forms of heart disease

\begin{tabular}{|c|c|c|c|c|}
\hline & $\begin{array}{l}\text { Sensitivity } \\
\text { (\%) }\end{array}$ & $\begin{array}{l}\text { Specificity } \\
(\%)\end{array}$ & $\begin{array}{l}\text { Area under } \\
\text { ROC }\end{array}$ & $95 \% \mathrm{Cl}$ \\
\hline Previous myocardial infarction & 100 & 97 & 0.986 & 0.977 to 0.992 \\
\hline Lone atrial fibrillation or flutter & 100 & 94 & 0.970 & 0.958 to 0.979 \\
\hline Hypertensive heart disease & 100 & 89 & 0.976 & 0.965 to 0.984 \\
\hline Valvar heart disease & 100 & 83 & 0.960 & 0.948 to 0.972 \\
\hline Hypertrophic cardiomyopathy & 80 & 83 & 0.833 & 0.810 to 0.855 \\
\hline
\end{tabular}




\section{DISCUSSION}

The present study has shown that plasma BNP measurement is useful for the detection of various forms of heart disease, particularly precursor forms of heart failure such as previous myocardial infarction, lone atrial fibrillation or flutter, hypertensive heart disease, and valvar heart disease, all of which are prone to lead to overt heart failure and to cardiovascular morbid events.

McDonagh and colleagues ${ }^{11}$ showed that in 1252 patients in the MONICA (monitoring trends and determinants in cardiovascular disease) risk factor survey, measurement of plasma BNP was a cost effective method for distinguishing the 37 participants $(3.0 \%)$ with LV systolic dysfunction (LV ejection fraction $<30 \%$ ) from those with preserved LV systolic function. In that study, the overall accuracy of plasma BNP measurement for predicting LV contractile dysfunction was modestly high as determined by a large area under the ROC curve $(>0.8)$ constructed by sensitivity and specificity at various cutoff levels. In contrast, Hetmanski and colleagues ${ }^{15}$ recently reported a poor overall accuracy. In a sample of 653 elderly patients currently prescribed loop diuretics for heart failure treatment, the area under the ROC curve was $<0.6$ for the detection of 314 participants $(48.1 \%)$ with an LV ejection fraction $<40 \%$. Their study suggests that plasma BNP measurement has relatively low sensitivity and specificity for detecting reduced LV systolic function. One of the important reasons for the discrepancy between these previous studies may be the difference in subjects' clinical background: mildly depressed LV systolic function is occasionally complicated by diastolic dysfunction as well as LV volume overload. In these pathophysiological conditions, plasma BNP concentrations are increased, even in the absence of any significant impairment in LV systolic function. Furthermore, circulating BNP concentrations are also raised in patients with hypertensive cardiac hypertrophy ${ }^{9}$ and in those with valvar heart disease, ${ }^{4}$ in whom LV contractile function is usually preserved. When considerable numbers of these subjects are included in a study group, the specificity and diagnostic ability to find subjects with LV systolic dysfunction are probably decreased.

Given the above limitations inherent in the use of plasma BNP measurement to identify impaired LV systolic function in a mass screening context, the present study examined the usefulness of this blood test for simply detecting various forms of heart disease irrespective of the degree of LV systolic dysfunction. Our findings in this instance were consistent with our previous study, ${ }^{19}$ with the finding that this test was especially effective for detecting subjects with a wide range of important cardiac abnormalities that would be amenable to early treatment.

There is considerable merit in finding forms of heart disease in a mass screening context. Increased plasma BNP concentrations has been shown to be a poor marker for long term prognosis independent of $\mathrm{LV}$ ejection fraction in patients with myocardial infarction. ${ }^{23}$ A population survey has suggested that $20-60 \%$ of subjects with non-fatal myocardial infarction are asymptomatic or unrecognised. ${ }^{24}$ Wolf and colleagues ${ }^{25}$ showed that the rate of stroke in patients with lone atrial fibrillation was more than five times as frequent as that in age, sex, and blood pressure adjusted populations. As anticoagulant treatment with warfarin reduces the relative risk more than $60 \%,{ }^{26}$ detection of these patients in the community by this blood test without time consuming ECG testing may save medical resources. Hypertensive LV hypertrophy is known to be a strong risk factor for future morbid events independent of age, blood pressure, or LV function. ${ }^{27}$ Intensive blood pressure lowering treatment has been reported to reverse LV hypertrophy and decrease plasma BNP concentrations in patients with essential hypertension. ${ }^{28}$ Use of BNP measurement to select patients with essential hypertension and LV hypertrophy for intensive antihypertensive treatment may be expected to reduce future cardiovascular events and improve prognosis. ${ }^{29}$ Identification of hypertrophic cardiomyopathy is also important. Although the overall sudden death rate is relatively low in this disorder, patients with multiple risk factors such as increased LV wall thickness, non-sustained ventricular tachycardia, syncope, family history of sudden death, and abnormal blood pressure response to exercise have been reported to have substantially increased risk of sudden death. ${ }^{30}$ Reports have suggested that either amiodarone or implantable cardioverter defibrillators may reduce the risk of sudden death in these high risk patients. ${ }^{31} 32$

No subjects showed impaired LV systolic function in the population studied here. McDonagh and colleagues ${ }^{33}$ reported a prevalence of severe LV systolic dysfunction as determined echocardiographically of $2.9 \%$ in an urban Scottish population. The predominant aetiology in this instance was ischaemic heart disease. Although no studies have examined the prevalence of LV systolic dysfunction in a general Japanese population, the large differences in prevalence of LV systolic dysfunction between the two populations may be caused by variation in the prevalence of coronary heart disease between Japan and Scotland..$^{34}$

There are no established blood tests for the detection of a wide range of heart conditions within large populations. Conventional methods such as medical interview, physical examination, chest radiography, ECG, and further echocardiography may consume considerable medical resources. The feasibility of further cardiac examination in cases with mild ECG abnormalities depends on physicians' decisions because no objective markers to assist the decision are yet available. In the present model, if all subjects showing abnormal ECG (table 1) had undergone more detailed examination by echocardiography, the total cost would have been approximately $£ 26700$, being $£ 8700$ for ECG ( $£ 8$ per single ECG) plus $£ 18000$ echocardiogram ( $£ 60$ per single echocardiogram). However, if plasma BNP measurement ( $>50 \mathrm{pg} / \mathrm{ml}$ ) had been used as an objective marker to determine which patients should undergo more detailed cardiac examination by ECG and echocardiography, the overall cost of screening, including the BNP test, would have been approximately half that of the ordinary screening procedure. This is based on a cost of $£ 8700$ for BNP measurement ( $£ 8$ per single BNP test). In this study 84 subjects would have been eligible for further cardiac examination by virtue of showing a BNP concentration above the cutoff value.

In conclusion, this study suggests that measurement of plasma BNP concentrations is an efficient and cost effective mass screening means of identifying patients with various forms of cardiac abnormality, regardless of aetiology and degree of LV systolic dysfunction, that can potentially develop into obvious heart failure and carry a high risk of cardiovascular events.

\section{ACKNOWLEDGEMENT}

We are grateful to Dr K Koyamada (IHMA) for his helpful suggestions for this study. We also thank the echocardiography laboratory staff at IHMA for their technical support.

\section{Authors' affiliations \\ M Nakamura, H Endo, M Nasu, N Arakawa, T Segawa, K \\ Hiramori, Second Department of Medicine, Iwate Medical University, Morioka, Japan}

\section{REFERENCES}

1 Sharpe N, Doughty R. Epidemiology of heart failure and ventricular dysfunction. Lancet 1998;352(suppl 1):SI3-7

2 Maclntyre K, Capewell S, Stewart S, et al. Evidence of improving prognosis in heart failure: trends in case fatality in 66547 patients hospitalized between 1986 and 1995. Circulation 2000;102:1126-31.

3 Firth BG, Yancy CW. Survival in congestive heart failure: have we made a difference? Am J Med 1990;88:3N-8N. 
4 Nakamura $M$, Niinuma $H$, Chiba $M$, et al. Effect of the maze procedure for atrial fibrillation on atrial and brain natriuretic peptide. Am J Cardiol 1997:79:966-70.

5 Richards AM, Crozier IG, Yandle TG, et al. Brain natriuretic factor: regional plasma concentrations and correlations with haemodynamic state in cardiac disease. Br Heart J 1993;69:414-7

6 Yasue $\mathbf{H}$, Yoshimura M, Sumida H, et al. Localization and mechanism of secretion of B-type natriuretic peptide in comparison with those of A-type natriuretic peptide in normal subjects and patients with heart failure. Circulation 1994;90:195-203.

7 Hasegawa K, Fujiwara H, Doyama K, et al. Ventricular expression of brain natriuretic peptide in hypertrophic cardiomyopathy. Circulation 1993:88:372-80.

8 Nishigaki K, Tomita M, Kagawa K, et al. Marked expression of plasma brain natriuretic peptide is a special feature of hypertrophic obstructive cardiomyopathy. J Am Coll Cardiol 1996;28:1234-42

9 Kohno M, Horio T, Yokokawa K, et al. Brain natriuretic peptide as a cardiac hormone in essential hypertension. Am J Med 1992;92:29-34.

10 Rossi A, Enriquez-Sarano M, Burnett JC, et al. Natriuretic peptide levels in atrial fibrillation: a prospective hormonal and Doppler-echocardiographic study. J Am Coll Cardiol 2000;35:1256-62.

11 McDonagh TA, Robb SD, Murdoch DR, et al. Biochemical detection of left-ventricular systolic dysfunction. Lancet 1998;351:9-13.

12 Smith H, Pickering RM, Struthers A, et al. Biochemical diagnosis of ventricular dysfunction in elderly patients in general practice: observational study. BM 2000;320:906-8.

13 Omland T, Aakvaag A, Vik-Mo H. Plasma cardiac natriuretic peptide determination as a screening test for the detection of patients with mild left ventricular impairment. Heart 1996;76:232-7

14 Davidson NC, Naas AA, Hanson JK, et al. Comparison of atrial natriuretic peptide B-type natriuretic peptide, and $\mathrm{N}$-terminal proatrial natriuretic peptide as indicators of left ventricular systolic dysfunction. Am J Cardiol 1996:77:828-31.

15 Hetmanski DJ, Sparrow NJ, Curtis S, et al. Failure of plasma brain natriuretic peptide to identify left ventricular systolic dysfunction in the community. Heart 2000;84:440-1.

16 McClure SJ, Caruana L, Davie AP, et al. Cohort study of plasma natriuretic peptides for identifying left ventricular systolic dysfunction in primary care. BM 1998;317:516-9.

17 Luchner A, Burnett JC, Jougasaki M, et al. Evaluation of brain natriuretic peptide as marker of left ventricular dysfunction and hypertrophy in the population. J Hypertens 2000;18:1121-8.

18 Omland T. Biochemical detection of systolic dysfunction. Lancet 1998;351:1063

19 Niinuma H, Nakamura M, Hiramori K. Plasma B-type natriuretic peptide measurement in a multiphasic health screening program. Cardiology 1998:90:89-94.
20 Sahn DJ, DeMaria A, Kisslo J, et al. Recommendations regarding quantitation in M-mode echocardiography: results of a survey of echocardiographic measurements. Circulation 1978:58:1072-83.

21 Teichholz LE, Kreulen T, Herman MV, et al. Problems in echocardiographic volume determinations: echocardiographic-angiographic correlations in the presence of absence of asynergy. Am J Cardiol 1976:37:7-11.

22 Imai Y, Tsuji I, Nagai K, et al. Ambulatory blood pressure monitoring in evaluating the prevalence of hypertension in adults in Ohasama, a rural Japanese community. Hypertens Res 1996;19:207-12.

23 Arakawa N, Nakamura M, Aoki $\mathrm{H}$, et al. Plasma brain natriuretic peptide concentrations predict survival after acute myocardial infarction. J Am Coll Cardiol 1996;27:1656-61.

24 Margolis JR, Kannel WS, Feinleib M, et al. Clinical features of unrecognized myocardial infarction: silent and symptomatic. Eighteen year follow-up: the Framingham study. Am J Cardiol 1973;32:1-7.

25 Wolf PA, Dawber TR, Thomas HE, et al. Epidemiologic assessment of chronic atrial fibrillation and risk of stroke: the Framingham study. Neurology 1978;28:973-7.

26 Singer DE. Anticoagulation to prevent stroke in atrial fibrillation and its implications for managed care. Am J Cardiol 1998;81:35C-40C.

27 Casale PN, Devereux RB, Milner M, et al. Value of echocardiographic measurement of left ventricular mass in predicting cardiovascular morbid events in hypertensive men. Ann Intern Med 1986;105:173-8.

28 Kohno M, Horio T, Yokokawa K, et al. Brain natriuretic peptide as a marker for hypertensive left ventricular hypertrophy: changes during 1-year antihypertensive therapy with angiotensin-converting enzyme inhibitor. Am J Med 1995;98:257-65.

29 Muiesan ML, Salvetti M, Rizzoni D, et al. Association of change in left ventricular mass with prognosis during long-term antihypertensive treatment. J Hypertens 1995;13:1091-5.

30 Elliott PM, Poloniecki J, Dickie S, et al. Sudden death in hypertrophic cardiomyopathy: identification of high risk patients. J Am Coll Cardiol 2000;36:2212-8

31 McKenna WJ, Oakley CM, Krikler DM, et al. Improved survival with amiodarone in patients with hypertrophic cardiomyopathy and ventricular tachycardia. Br Heart J 1985:53:412-6.

32 Maron BJ, Shen WK, Link MS, et al. Efficacy of implantable cardioverter-defibrillators for the prevention of sudden death in patients with hypertrophic cardiomyopathy. N Engl J Med 2000;342:365-73.

33 McDonagh TA, Morrison CE, Lawrence A, et al. Symptomatic and asymptomatic left-ventricular systolic dysfunction in an urban population. Lancet 1997;350:829-33.

34 Marmot MG. Life style and national and international trends in coronary heart disease mortality. Postgrad Med J 1984;60:3-8.

\section{IMAGES IN CARDIOLOGY}

\section{Embolism of an idiopathic pulmonary artery aneurysm}

$A^{s}$

56 year old woman was referred to our hospital with a 12 month history of exertional breathlessness. Auscultation revealed a soft ejection systolic murmur maximal at the left sternal edge. Electrocardiography and echocardiography were normal. Chest radiography revealed a well defined $1.5 \mathrm{~cm}$ diameter spherical mass adjacent to the left atrial appendage (left panel). Selective left pulmonary angiography confirmed a pulmonary artery aneurysm (PAA) arising from the lingular artery (right panel). The feeding artery was embolised with coils (Cook UK) and angiography confirmed successful exclusion of the aneurysm from the circulation.

PAAs are a rare abnormality. Deterling and Claggett noted the vascular anomaly in only eight of a series of 100000 unselected necropsies and over $50 \%$ of cases were associated with congenital heart disease. Patients with PAA may be entirely asymptomatic or complain of symptoms, such as chest pain, cough, and breathlessness. Little is known about the natural history of PAAs; however, all aneurysms have the potential for rupture and it has been suggested that a third of patients with proximal PAA die from such a haemorrhage. We safely and effec-
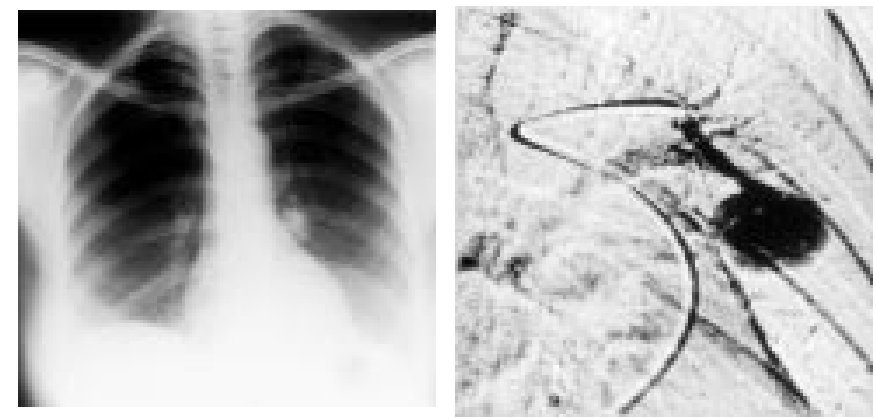

P Mann

D Seriki

P A Dodds

paul1man@aol.com tively treated a peripheral anomaly with coil embolisation. 